\title{
Block-Spin Approach to Electron Correlations
}

\author{
P. Monthoux \\ National High Magnetic Field Laboratory and Department of Physics \\ Florida State University, Tallahassee, Florida 32306
}

(November 20, 2016)

\begin{abstract}
We consider an expansion of the ground state wavefunction of quantum lattice many-body systems in a basis whose states are tensor products of block-spin wavefunctions. We demonstrate by applying the method to the antiferromagnetic spin- $1 / 2$ Heisenberg chain that by selecting the most important many-body states the technique affords a severe truncation of the Hilbert space while maintaining high accuracy.
\end{abstract}

PACS Nos. 71.10.+x, 75.27.-b 


\section{INTRODUCTION}

The basic physical idea underlying the Renormalization Group (RG) approach to critical phenomena and quantum field theory is that the many length or energy scales are locally coupled. There is then a cascade effect in the whole system: the eV energy scales influence the $100 \mathrm{meV}$ energy scales. The $100 \mathrm{meV}$ scales influence the $10 \mathrm{meV}$ energy scales, etc. The RG formalism can then be set up to reduce the problem into one involving a finite number of degrees of freedom at each iterationst.

Wilson's solution of the Kondo problem 1 by means of RG techniques inspired many attempts at a RG approach to quantum lattice model\$2 2 . These latter real space RG schemes employed a Kadanoff block-spin construction. The lattice is partitioned into blocks of a few sites each and the Hamiltonian on a block is diagonalized. One seeks an effective Hamiltonian that describes the physics of the low energy degrees of freedom in each block. The contribution of the high energy states of the block is either ignored altogether in the simplest approximation or incorporated by low order perturbation theory. While generally good results were obtained for the ground state state energy of the system, the ground state wavefunction was not satisfactorily described. These simple effective Hamiltonian approaches suffer from small energy denominators, e.g. the denominators involving differences of the highest energy kept minus the lowest energy eliminated 3 .

The Density Matrix Renormalization Group (DMRG) algorithm solved the problems that plagued the simple real-space RG schemes with two clever ideas. Instead of doubling the system size at each RG iteration, one adds one site to the system at each iteration. The wavefunctions of the N-site and N+1-site systems should be "close" to each other, thus allowing a truncation of the Hilbert space without much loss in accuracy. The second idea is to use the eigenstates of the block density matrix as a basis instead of the eigenstates of

the Hamiltonian. In two insightful papers, Rommer and Ostlund国 showed that in the case where the DMRG converges to a fixed point, the quantum states in the thermodynamic limit with periodic boundary conditions can be simply represented by a "matrix product 
ground state". These states can be obtained through a simple variational ansatz, in the construction of which the DMRG plays no essential role, aside from providing a guide to which representations to keep. Such "matrix product states" are in fact exact ground states for some particular 1-d models 6 . One can almost certainly attribute the success of the DMRG method in 1-d to the clever choice of a correlated basis that describes the physics of such systems so well.

By the same token, one can speculate that the difficulties encountered in tackling 2-d problems with the DMRG method may be due to the fact that the "matrix product states" are not so well tuned to the physics of 2-d systems, which is quite different from that of their 1-d counterparts. It is usually advisable to use a symmetry-adapted basis with as many symmetries of the eigenstates one is interested in as possible. But any such matrix product state in 2-d cannot be translation invariant and does not transform according to any of the irreducible representations of the symmetry group of the lattice. This must limit the accuracy achievable in 2-d with a matrix product ansatz for the ground state. In the present work, a different correlated basis is used to expand the many-body wavefunction that can be used (in principle) in any dimension.

The main difficulty in carrying out the RG program for a quantum many-body Hamiltonian is to find a representation of the problem (basis) for which the assumption of locally coupled energy scales is valid, at least to a good approximation. We have carried out simple numerical experiments on random matrices 10 and we found that for the many energy scales to be locally coupled, the Hamiltonian matrix elements $H_{i, j}$ have to decay away from the diagonal. If the decay follows a power law (say $H_{i, j}$ goes like $1 /\left|E_{i}-E_{j}\right|$ ), the weights of the basis states in the ground state wavefunction decay (on average) as the inverse square of their energy. If the decay of the matrix elements away from the diagonal is exponential, then the weights of the basis states in the ground state wavefunction decay (on average) exponentially with energy. In either case, one has a well defined hierarchy of basis states, and one can envisage solving the problem by means of $R G$ techniques. A renormalization procedure for Hamiltonians such as those in light-front field theory that achieves this lo- 
cal coupling of energy scales while avoiding the small denominator problem is presented in Ref.․ The bare Hamiltonian is transformed by a particular similarity transformation. This transformation is such that all matrix elements vanish which would have otherwise caused transitions with big energy jumps.

If one diagonalizes a strongly correlated system, such as the 24-site spin-1/2 Heisenberg chain in an uncorrelated basis (tensor product of single site states), one finds that at energies five or six times the exchange constant above the mean-field (or Néel) state, the weights of the basis states of equal energy span six to eight orders of magnitude! Such a basis is useless for carrying out the RG program.

A suitable basis must therefore include some of the correlations present in the true many-body ground state. It must remain simple enough, however, so that the evaluation of matrix elements of the Hamiltonian can be done in a resonable amount of computer time, and thus a good compromise must be found. In this paper, we examine a block-spin basis for expanding the wavefunction of quantum lattice models with short-range interactions. Since the energy essentially depends on correlations on a scale of the range of the interactions, it is dominated by short-range correlations. Such correlations can be taken into account by grouping the sites into blocks of a few sites each and treating the interactions among the sites in a given block non-perturbatively. There are of course other ways of taking into account short-range correlations, but the advantage of the block-spin construction is that is provides an orthonormal basis and the evaluation of matrix elements of the Hamiltonian is straighforward. The long-range correlations, i.e. correlations between sites in different blocks, can be taken into account by using a linear superposition of tensor products of blockspin states. For large systems, it is impossible in practice to retain all such tensor products, and one must truncate the expansion to the most important many-body configurations. We thus make use of the adaptive state selection techniques used in the context of Quantum Chemistry 1 . We demonstrate the method by applying it to a test problem whose exact solution is known, the 40-site antiferromagnetic spin-1/2 Heisenberg chain. We find that it is possible to recover $99.8 \%$ of the correlation energy and to describe the spin correlations at 
a distance of 20 lattice spacings with an accuracy of $1.5 \%$ with only $2 \times 10^{-4} \%$ of the Hilbert space, a problem that can be solved on a personal computer. In the following section, we present the model and describe the block-spin basis. We then discuss the adaptive state selection algorithm employed in this work and present our results. In the final section, we examine the strengths and weaknesses of the approach and discuss its possible generalizations and extensions.

\section{BLOCK-SPIN BASIS}

We wish to illustrate the method on a simple model problem, the 40-site Heisenberg spin $\frac{1}{2}$ chain with periodic boundary conditions. The Hamiltonian is

$$
\mathrm{H}=\mathrm{J} \sum_{\mathrm{i}=1}^{\mathrm{N}}\left\{\mathrm{S}_{\mathrm{i}}^{\mathrm{z}} \mathrm{S}_{\mathrm{i}+1}^{\mathrm{z}}+\frac{1}{2}\left(\mathrm{~S}_{\mathrm{i}}^{+} \mathrm{S}_{\mathrm{i}+1}^{-}+\mathrm{S}_{\mathrm{i}}^{-} \mathrm{S}_{\mathrm{i}+1}^{+}\right)\right\}
$$

where $\mathrm{N}=40$ and $\mathrm{S}_{\mathrm{N}+1}^{\alpha} \equiv \mathrm{S}_{1}^{\alpha}, \alpha=z, \pm$. In the following we set the exchange constant J $=1$. We group the sites in blocks of 8 spins and consider the following tensor products of block-spin states

$$
\left|b_{i}^{(1)}>\bigotimes\right| b_{j}^{(2)}>\bigotimes\left|b_{k}^{(3)}>\bigotimes\right| b_{l}^{(4)}>\bigotimes \mid b_{m}^{(5)}>
$$

where the block states $\left\{\mid b_{\mathrm{p}}^{(\mathrm{m})}>\right\}$ are linear combinations of the Ising basis states on each block:

$$
\left|\mathrm{b}_{\mathrm{p}}^{(\mathrm{m})}>=\sum_{\{\sigma\}} \mathrm{C}^{(\mathrm{p})}\left(\sigma_{8(\mathrm{~m}-1)}, \sigma_{8(\mathrm{~m}-1)+1}, \ldots, \sigma_{8 \mathrm{~m}}\right)\right| \sigma_{8(\mathrm{~m}-1)}, \sigma_{8(\mathrm{~m}-1)+1}, \ldots, \sigma_{8 \mathrm{~m}}>
$$

where $\sigma_{i}=\uparrow, \downarrow$ is the $\mathrm{S}_{\mathrm{z}}$ component of the spin on site $\mathrm{i}$, and $\left\{\mathrm{C}^{(\mathrm{p})}\right\}_{\mathrm{p}=1}^{2^{8}}$ are real numbers such that the states $\left\{\mid \mathrm{b}_{\mathrm{p}}^{(\mathrm{m})}>\right\}_{\mathrm{p}=1}^{2^{8}}$ form an orthonormal basis for block $\mathrm{m}$. Since the ground state is translationally invariant, all the blocks are equivalent and thus the many-body states that can be obtained by a cyclic permutation of the block indices will have the same coefficient in the wavefunction. We use this symmetry along with the conservation of the total $\mathrm{S}_{\mathrm{z}}^{\text {tot }}$ component of the $\mathrm{z}$-axis magnetization $\left(\mathrm{S}_{\mathrm{z}}^{\text {tot }}=0\right.$ for the ground state $)$ to reduce the 
dimension of the matrix that must be diagonalized. Even with these symmetries taken into account, the Hilbert space in the ground state symmetry sector contains approximately 20 billion states. This number is larger than the size of the matrix one would have to diagonalize had one used the simple Ising basis of tensor products of single site states. The reason is that we have only taken into account translation invariance at the block scale, and thus only reduces the dimension of the Hilbert space by a factor $\sim 5$ instead of the usual factor $\sim \mathrm{N}$ when the full translation invariance is taken into account. If one were to construct Bloch states out of the tensor products, Eq.( 2), the basis would no longer be orthonormal and the evaluation of the matrix elements of the Hamiltonian and the calculation of the overlap matrix would be much more complicated.

All that is required of the coefficients $\left\{\mathrm{C}^{(\mathrm{p})}\right\}_{\mathrm{p}=1}^{2^{8}}$ is that they provide a orthonormal basis for a block-spin. One therefore has some freedom in choosing the block states, and this should clearly be used to one's advantage. A drawback of the block-spin construction is that it introduces artificial boundaries between blocks in the system. The exact solution does not of course depend on the basis, but since one has to trunctate the expansion of the wavefunction, there will be some effect of the block boundaries and one ought to minimize it. To this end let us choose the states on the blocks to be the eigenstates of the Hamiltonian

$$
\mathcal{H}_{\text {block }}=\sum_{\mathrm{i}, \mathrm{i}+1 \in \text { block }} \mathrm{J}_{\mathrm{i}} \overrightarrow{\mathrm{S}}_{\mathrm{i}} \cdot \overrightarrow{\mathrm{S}}_{\mathrm{i}+1}
$$

where the coupling constants $\left\{\mathrm{J}_{\mathrm{i}}\right\}$ are taken as parameters. The block-spin basis clearly depends on the $\left\{\mathrm{J}_{\mathrm{i}}\right\}$ and one can use the variational principle to set these parameters to values such that the expectation value of the full Hamiltonian with respect to some trial wavefunction is minimized. We have found by calculating the eigenstates of the ground state block density matrix for the 24-site chain that the probability of finding the block in the lowest singlet or triplet state is roughly $90 \%$. Thus we use as the trial wavefunction all of the possible many-body states with $\mathrm{S}_{\mathrm{z}}^{\text {tot }}=0$ that can be formed with the lowest singlet and triplet states in each block. For the 40-site chain, there are 52 such configurations, taking into account the equivalence of the blocks. We have found that taking more states of each 
block into account improves the results only very slightly while increasing the computational cost considerably. This extra computer time is much better spent on the adaptive selection of the many-block configurations described in the next section. Carrying out the optimization with the 52 configurations, one obtains the following values for $\left\{\mathrm{J}_{\mathrm{i}}\right\}$ :

$$
\mathrm{J}_{1}=\mathrm{J}_{7}=0.33 ; \quad \mathrm{J}_{2}=\mathrm{J}_{6}=0.70 ; \quad \mathrm{J}_{3}=\mathrm{J}_{5}=0.81 ; \quad \mathrm{J}_{4}=1.00
$$

The result of this optimization is to make the ends of the blocks softer, allowing them to better adapt to the presence of their neighbors. Such smooth boundary conditions were

introduced by Vekic and White 2 in order to minimize finite size effects. They were also shown by the same authors to considerably improve the "fermion sign problem" in Quantum Monte Carlo simulations 3 .

\section{TRUNCATION OF MANY-BODY WAVEFUNCTION}

Since in practice it is not going to be possible to keep all of the possible tensor products of block-spin states with the proper quantum numbers, we would like to select the most important ones, namely the many-body states with the largest weights in the ground state wavefunction. Consider a complete orthonormal basis $\left\{\mid \phi_{\mathrm{n}}>\right\}$. The ground state wavefunction of the many-body system $\mid \psi_{0}>$ can (at least in principle) be expanded as

$$
\left|\psi_{0}>=\sum_{\mathrm{n}} \mathrm{c}_{\mathrm{n}}\right| \phi_{\mathrm{n}}>
$$

We would like to truncate the expansion, Eq.( [6) such that all of the coefficients $\left|c_{n}\right|$ are larger than some threshold $\epsilon$. The other many-body states are discarded. The results become exact as $\epsilon \rightarrow 0$. The algorithm to pick the set of configurations such that $\epsilon<\left|\mathrm{c}_{\mathrm{n}}\right|$ must be adaptive, since the states that one may wish to add to our set depend on what states we have already kept. For instance, there is no point in adding a state $\mid \phi_{\mathrm{i}}>$ with $\left\langle\phi_{\mathrm{i}}|\mathrm{H}| \phi_{\mathrm{n}}>=0\right.$ for all $\mid \phi_{\mathrm{n}}$ >'s in our set, since upon diagonalizing the Hamiltonian in that restricted subspace, the coefficient of $\mid \phi_{\mathrm{i}}>$ in the approximate ground state wavefunction vanishes. 
Let us change the notation and denote $\{\mid \alpha>\}$ the set of $\mathrm{N}_{\mathrm{s}}$ configurations retained (internal space), $\mid \psi^{(0)}>$ the variational ground state in the $\{\mid \alpha>\}$ subspace of energy $\mathrm{E}^{(0)}$, and $\{\mid \beta>\}$ the set of states not retained (external space). We first estimate the correction $\mid \delta \psi>$ from the external space to the approximate eigenstate $\mid \psi^{(0)}>$ by first order perturbation theory, using as unperturbed Hamiltonian

$$
\tilde{\mathrm{H}}_{0}=\left|\psi^{(0)}>\mathrm{E}^{(0)}<\psi^{(0)}\right|
$$

and

$$
\tilde{\mathrm{H}}_{1}=\mathrm{H}-\tilde{\mathrm{H}}_{0}
$$

as the perturbation. One thus has

$$
\begin{gathered}
\left|\psi^{(0)}>\equiv \sum_{\alpha} \mathrm{c}_{\alpha}\right| \alpha> \\
\left|\delta \psi>\equiv \sum_{\beta} \mathrm{c}_{\beta}\right| \beta>
\end{gathered}
$$

where the coefficients $\left\{\mathrm{c}_{\alpha}\right\}$ where obtained by diagonalizing the Hamiltonian in the internal space and we wish to estimate the coefficients $\left\{\mathrm{c}_{\beta}\right\}$. To first order in $\tilde{\mathrm{H}}_{1}$, one has

$$
\mathrm{c}_{\beta}=\frac{1}{\mathrm{E}^{(0)}} \sum_{\alpha} \mathrm{c}_{\alpha}<\alpha|\mathrm{H}| \beta>
$$

At this stage, we discard the configurations for which $\left|\mathrm{c}_{\beta}\right|<\epsilon^{\prime}<\epsilon$ (in practice $\epsilon^{\prime}=0.5 \epsilon$ works fine). The rationale for estimating the correction to $\left|\psi^{(0)}\right\rangle$ by perturbation theory first is that one would like to identify the configurations that have weights much smaller than the threshold and eliminate those right away. This way the internal space does not expand too much at each iteration, the dimension of the matrix that one must diagonalize is significantly smaller and it saves a significant amount of computer time. On the other hand, since we only estimate the coefficients to first order, we are making some error and in the event the coefficients of some states are underestimated in the calculation, one might throw away states that should be kept. The present method compromises by keeping states 
with coefficients slightly smaller in magnitude than the final cutoff. It is found in practice that the error introduced by this early truncation is order of magnitudes smaller than the difference between the variational energy and the exact result (typically in the sixth digit). It is therefore perfectly legitimate and speeds up the computation by at least one order of magnitude. Next, we diagonalize the Hamiltonian matrix in the expanded internal space and the states whose coefficients $\mathrm{c}_{\alpha}$ are smaller in magnitude than the threshold $\epsilon$ are discarded. The procedure can be repeated a number of times, until no more states whose coefficients are larger in magnitude than $\epsilon$ can be added to the set.

This method works very well for any type of basis. However, there is one feature of the block-spin basis that requires a couple more tricks. In the basis that consists of tensor products of single spin states (Ising basis), the Hamiltonian acting on a state can produce at most $\mathrm{N}$ (the number of sites) other states, since there are N spin-flip terms in the Hamiltonian, Eq.(1). Since the total number of states grows exponentially with N, the matrix representation of the Hamiltonian in this simple basis is extremely sparse. The block-spin states are linear combinations of many Ising basis states (up to 70 in the $S_{z}=0$ sector of the eight spin block) and thus each member of the many-body basis of tensor products of block-spin states contains a very large number of Ising basis states. The matrix representation of the Hamiltonian in this block-spin basis is not very sparse and the application of the Hamiltonian operator to any internal state $\mid \alpha>$ produces a very large number of external states $\mid \beta>$. If the Hamiltonian matrix were full, one could generate the entire external space by applying the Hamiltonian to a single state. If the matrix is not completely full, there is still some redundancy and it is not necessary to apply the Hamiltonian to every state to generate the external space (of course in general we do not know which subset of states will generate the complete external space). We also expect that the next most important states are those that directly couple to the most important states we already have (that actually depends on the quality of the basis, i.e. the smallness of off-diagonal matrix elements of the Hamiltonian for states far apart in energy). We have used these heuristics to modify the algorithm as follows: one generates the states $\mid \beta>$ in the external space by applying 
the Hamiltonian only to the most important states in the internal space, namely those with coefficients larger in magnitude than $\epsilon ">\epsilon$. In practice, one must test various values of $\epsilon$ " to determine a good compromise between accuracy and speed of computation. We have found that it is possible to choose $\epsilon$ " such that the error introduced is much smaller than the error due to the truncation of the Hilbert space. Another simplification is to restrict a priori the number of block states that are to be used. In the early steps of the algorithm, there is no need to consider very highly excited states of the blocks. This restriction can be loosened as the adaptive state selection proceeds. One can also do that in such a way that the approximation introduces an error less than the systematic error due to the truncation of the many-body wavefunction. These tricks speed up the algorithm by at least an order of magnitude. It is of course possible to relax each of these approximations to estimate the effect they have on the final results.

By studying the increase in the number of states kept and the distribution of the weights of these states as a function of their energy in the variational ground state wavefunction as the cutoff $\epsilon$ is decreased, the adaptive state selection algorithm described above can give us a good idea of the quality of the basis used and how the off-diagonal matrix elements decrease as the energy difference between states increases.

\section{RESULTS}

It is important that the method not only give accurate results for local quantities such as the ground state energy, but it should also yield accurate results for the long-range spin correlations. The ground state spin-spin correlation function $\mathrm{C}(\mathrm{l})=<\overrightarrow{\mathrm{S}}(\mathrm{i}) \cdot \overrightarrow{\mathrm{S}}(\mathrm{i}+\mathrm{l})>$ is shown in Fig. 1 where it is compared to the Quantum Monte Carlo results of Sandvik 14. Since the method only takes into account the equivalence between blocks and not the full translation invariance, the results of this calculation would actually depend on the site $\mathrm{i}$. The quantity $\mathrm{C}(\mathrm{l})$ shown in Fig. 1 is averaged over i: 


$$
\mathrm{C}(\mathrm{l})=\frac{1}{\mathrm{~N}} \sum_{\mathrm{i}}<\overrightarrow{\mathrm{S}}(\mathrm{i}) \cdot \overrightarrow{\mathrm{S}}(\mathrm{i}+\mathrm{l})>
$$

In this calculation, the adaptive state selection algorithm described in the previous section was used with a cutoff $\epsilon=5 \times 10^{-4}$. The variational ground state wavefunction obtained is a linear superposition of 8553 (out of a possible 20 billion) basis states (therefore about $2 \times 10^{-4 \%}$ of the Hilbert space), and only 56 out of the 256 block basis states were actually used. The other parameters used in the calculation were $\epsilon^{\prime}=0.5 \epsilon$ and $\epsilon^{\prime \prime}=5 \times 10^{-3}$. The variational ground state energy obtained is $-17.728 \mathrm{~J}$ compared to the exact value of -17.7465227883 J from the Bethe ansatz国. Given the energy of the mean-field (Néel) state of -10 J, one recovers $99.8 \%$ of the correlation energy. One does a good job with the long-range spin correlations. Note that for a separation of 16 lattice sites and over, the spins are not even in nearest neighbor blocks. The error with respect to the Monte-Carlo results is about $1.5 \%$ for $\mathrm{C}(\mathrm{l}=20)$. It is instructive to look at what the spin-spin correlations look like in the block-spin mean-field ground state

$$
\left|\Psi_{\mathrm{MF}}>=\right| \mathrm{b}_{0}^{(1)}>\bigotimes\left|\mathrm{b}_{0}^{(2)}>\bigotimes\right| \mathrm{b}_{0}^{(3)}>\bigotimes\left|\mathrm{b}_{0}^{(4)}>\bigotimes\right| \mathrm{b}_{0}^{(5)}>
$$

with the smooth boundary conditions and with open boundary conditions. In the above equation $\mid b_{0}^{(i)}>$ is the ground state of block i. The results are shown in Fig. 2. For nearest neighbor spins (i.e. for the energy), the block-spin states with open boundary conditions give a lower variational energy. On the other hand, for correlations at larger distances, the block-spin states with the smooth boundary conditions do a much better job. Note that a single tensor product of block-spin states does not correlate spins in different blocks and thus the correlation function $\mathrm{C}(\mathrm{l})=0$ when $\mathrm{l} \geq 8$, as it should. If one uses the block-spin basis with open boundary conditions, one needs nearly ten times as many basis functions (approximately 80,000) to obtain results of quality similar to those shown in Fig. 1. It is thus worthwile to slightly increase the ground state energy of the block in order to better describe the spin-spin correlations at distances larger than one lattice spacing.

We have also looked at choosing the basis on a block in the spirit of the DMRG, namely by calculating the block density matrix from some approximate ground state wavefunction 
and using as the block basis the eigenstates of the block density matrix. One can of course iterate the process and recompute an approximate ground state wavefunction using the improved block basis, and using the eigenstates of the new block density matrix. The process converges in a few iterations. It turns out, however, that the block basis obtained in this way is very similar to the one one gets with the smooth boundary conditions, and leads to results of the same quality.

We have mentioned in the introduction that one would like to have a basis such that the importance of the states decreases as their energy increases. In Fig. 3 we show the absolute value of the coefficients of the 8553 basis states in the variational wavefunction used in the calculation of $\mathrm{C}(\mathrm{l})$ shown in Fig. 1 as a function of their energy. At low energy, the weights of the states decay roughly exponentially as a function of energy, but the situation totally deteriorates at higher energies, where there is no longer a clear hierarchy of the basis states. We speculate that this is due to the effect of the block boundaries. The basis states do not have all of the symmetries of the exact ground state, and this ultimately limits the accuracy achievable with this method. The smooth boundary conditions do not completely eliminate the problem. In order to obtain much more accurate results than those shown in Fig. 1, one would have to retain an exponentially larger number of states. As one can see from Fig. 3, for cutoffs $\epsilon$ much smaller than $5 \times 10^{-4}$, one would have to keep states in a wide energy range and since the density of states increases exponentially with energy, one needs to keep an exponentially larger number of states.

\section{OUTLOOK}

We have shown that an expansion of the ground state many-body wavefunction in terms of a block-spin basis allows a severe truncation of the Hilbert space while maintaining good accuracy for a 40-site Heisenberg spin-1/2 chain. For this problem, the number of basis states one must use (for a given accuracy) grows exponentially with the number of blocks in the system: spins in any two blocks, however far apart, must be correlated. Model 
Hamiltonians whose excitation spectrum possesses a gap in the thermodynamic limit do not of course present this difficulty. In the present case, however, this ultimately limits the size of the system that can be treated accurately. For a spin-1/2 system, it is not inconceivable to use blocks of 12 spins, and with five such blocks one should be able to tackle systems with up to 60 spins, keeping on the order of 10,000-20,000 many-block configurations. We have not attempted this, since it would have required an extensive reprogramming effort due to memory constraints on the computer system used for these calculations. Note that the DMRG method for this problem is superior, in that much larger systems can be treated with ease. The purpose of the present work was not an attempt to supersede the DMRG in one dimension (in the opinion of the author, this extremely unlikely to happen), but to study an alternative many-body basis in the hope that it could be used when the DMRG method is not as effective.

The block-spin approach is trivially extended to two dimensions. We have in fact carried out calculations for two-dimensional spin-1/2 Heisenberg systems. In higher dimensions, the quantum fluctuations are weaker than for the one-dimensional spin chain, and as a result less correlation energy must be recovered given a fixed number of spins. We therefore expected the two-dimensional problem to be easier to solve with the present technique. Unfortunately, it has turned out otherwise. The possibility of long-range order in two dimensions is at the root of the problem. Since the ground state of the two-dimensional Heisenberg model possesses long-range order, the correlations of spins in widely separated blocks are such that they saturate to a finite constant value as the separation between the blocks goes to infinity. On the other hand, our block-spin basis is such that spins in different blocks are completely uncorrelated. In one dimension, one can build up these block-block correlations with some effort, since they decay algebraically. Uncorrelated spins a distance 8 apart provides quite a good starting point. In two-dimensions, one has to start with uncorrelated spins a distance 3 or 4 apart $(3 \times 3$ or $4 \times 4$ are clearly the maximum block sizes that can be handled $)$ and it turns out to be a much poorer starting point. The possibility of long-range order in two-dimensions must therefore be incorporated in the basis in some way. We plan to look 
at various possibilities.

As the DMRG algorithm has demonstrated, computational methods based on an approximate diagonalization of the Hamiltonian can be competitive with Monte Carlo methods. The success of the DMRG and the results presented in this paper indicate that such an approximate diagonalization is only effective if carried out in a correlated basis that closely approximates the true many-body ground state correlations. The challenge is to find such a basis for the many two and three-dimensional model Hamiltonians which remain unsolved.

I should like to thank Z. Ha, E. Manousakis, and A. Sandvik for discussions on these and related topics. This work was supported by the National High Magnetic Field Laboratory and the State of Florida. 


\section{REFERENCES}

* Address after 1 October 1997: Cavendish Laboratory, University of Cambridge, Madingley Road, Cambridge, CB3 0HE, United Kingdom.

${ }^{1}$ K.G. Wilson, Rev. Mod. Phys. 47, 773 (1975).

${ }^{2}$ S. Drell, M. Weinstein, and S. Yankielowitcz, Phys. Rev. B 16, 1769 (1977) ; J. Hirsch, Phys. Rev. B 22, 5229 (1980) ; C. Dasgupta and P. Pfeuty, J. Phys. C 14, 717 (1981) ; T.P. Zivkovic, B.L. Sandenback, T.G. Schmalz, and D.L. Klein, Phys. Rev. B 41, 2249 (1990) ; M.B. Lepetit and E. Manousakis, Phys. Rev. B 48, 1028 (1993).

${ }^{3}$ S.D. Glazek, K.G. Wilson, Phys. Rev. D 49, 4214 (1994) ; Phys. Rev. D 48, 5863 (1993).

${ }^{4}$ S.R. White, Phys. Rev. B 48, 110345 (1993) ; Phys. Rev. Lett. 69, 2863 (1992).

${ }^{5}$ S. Rommer and S. Ostlund, Phys. Rev. B 55, 2164 (1997) ; Phys. Rev. Lett. 75, 3537 (1995).

${ }^{6}$ I. Affleck, T. Kennedy, E.H. Lieb, and H. Tasaki, Phys. Rev. Lett. 59, 799 (1987) ; Commun. Mth. Phys. 115, 477 (1988).

${ }^{7}$ L. Accardi, Phys. Rep. 77, 169 (1981).

${ }^{8}$ M. Fannes, B. Nachtergaele and R.F. Werner Europhys. Lett. 10, 633 (1989) ; Commun. Math. Phys. 144, 443 (1992).

${ }^{9}$ A. Klümper, A. Schadschneider, and J. Zittarz, Europhys. Lett. 24, 293 (1993) ; C. Lange, A. Klümper, and J. Zittarz, Z. Phys. B 96, 267 (1994).

${ }^{10}$ Z.N.C. Ha and P. Monthoux, unpublished.

${ }^{11}$ W. Wentzel and K.G. Wilson, Phys. Rev. Lett. 69, 800 (1992); M.M. Steiner, W. Wentzel, K.G. Wilson, J.W. Wilkins, Chem. Phys. Lett. 231, 263 (1994).

${ }^{12}$ M. Vekic and S.R. White, Phys. Rev. Lett. 71, 4283 (1993). 
${ }^{13}$ M. Vekic and S.R. White, Phys. Rev. B 53, 14552 (1996).

${ }^{14}$ A.W. Sandvik, private communication.

${ }^{15}$ Z.N.C. Ha, private communication. 


\section{FIGURES}

FIG. 1. The spin-spin correlation function, Eq. (12), with the adaptive state selection parameters $\epsilon=5 \times 10^{-4}, \epsilon^{\prime}=\epsilon / 2$ and $\epsilon^{\prime \prime}=5 \times 10^{-3}$ (diamonds) is compared to the Monte Carlo results of Sandvik 14 (dashed line and error bars).

FIG. 2. The spin-spin correlation function, Eq. (12), for the block mean-field state, Eq. (13), is shown with smooth boundary conditions on the blocks (diamonds) and with open boundary conditions on the blocks (crosses).

FIG. 3. Writing the variational ground state wavefunction $\left|\psi_{0}>=\sum_{\mathrm{i}} \mathrm{c}_{0}(\mathrm{i})\right| \phi_{\mathrm{i}}>$ in the basis $\left\{\left|\phi_{\mathrm{i}}\right\rangle\right\}$, we show the magnitude of the coefficients $c_{0}(\mathrm{i})$ for the block-spin basis state $\mid \phi_{\mathrm{i}}>$ as a function of its energy $<\phi_{\mathrm{i}}|\mathrm{H}| \phi_{\mathrm{i}}>$. The variational wavefunction used is obtained from the adaptive state selection algorithm with the same parameters as described in Fig. 1 and in the text. 


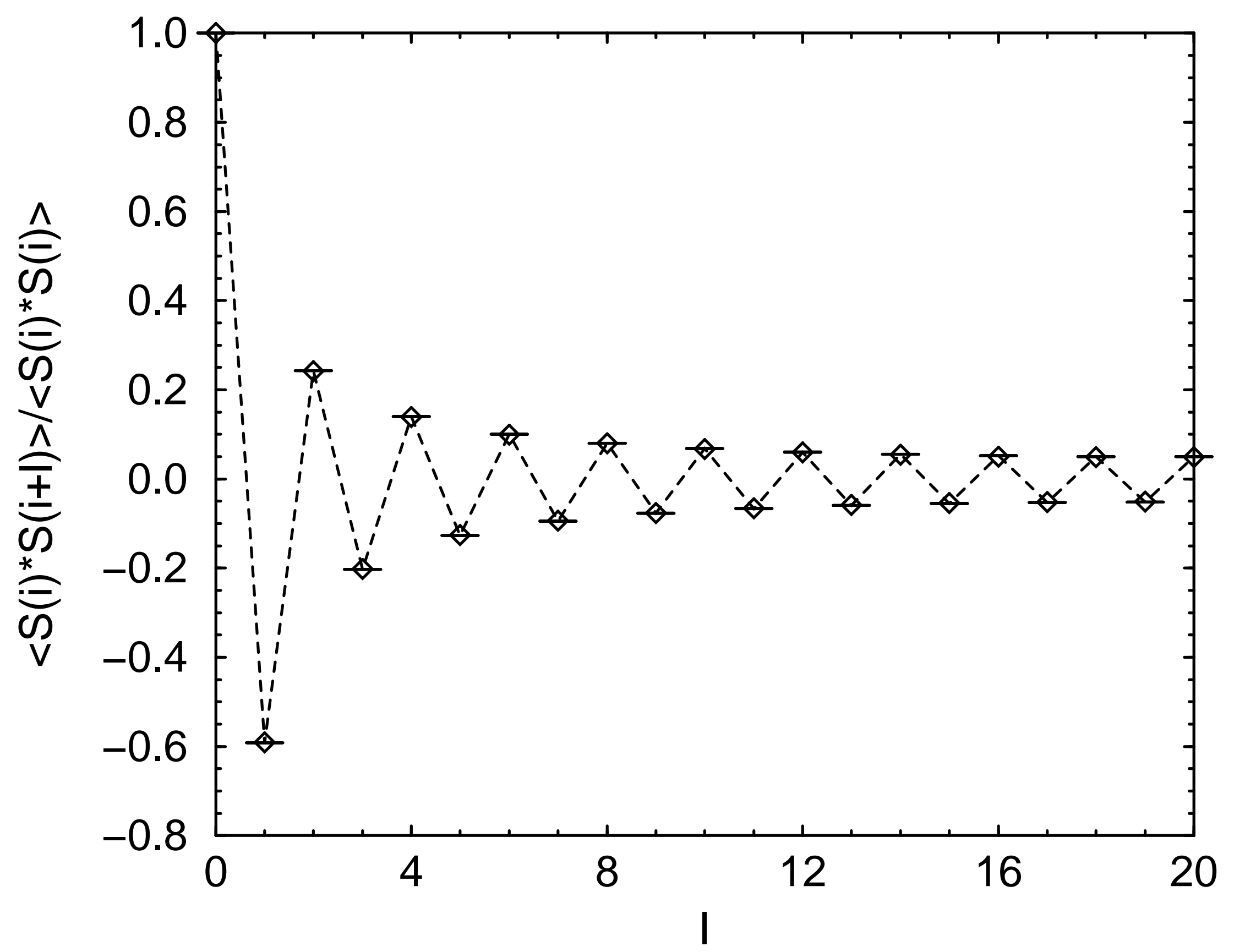




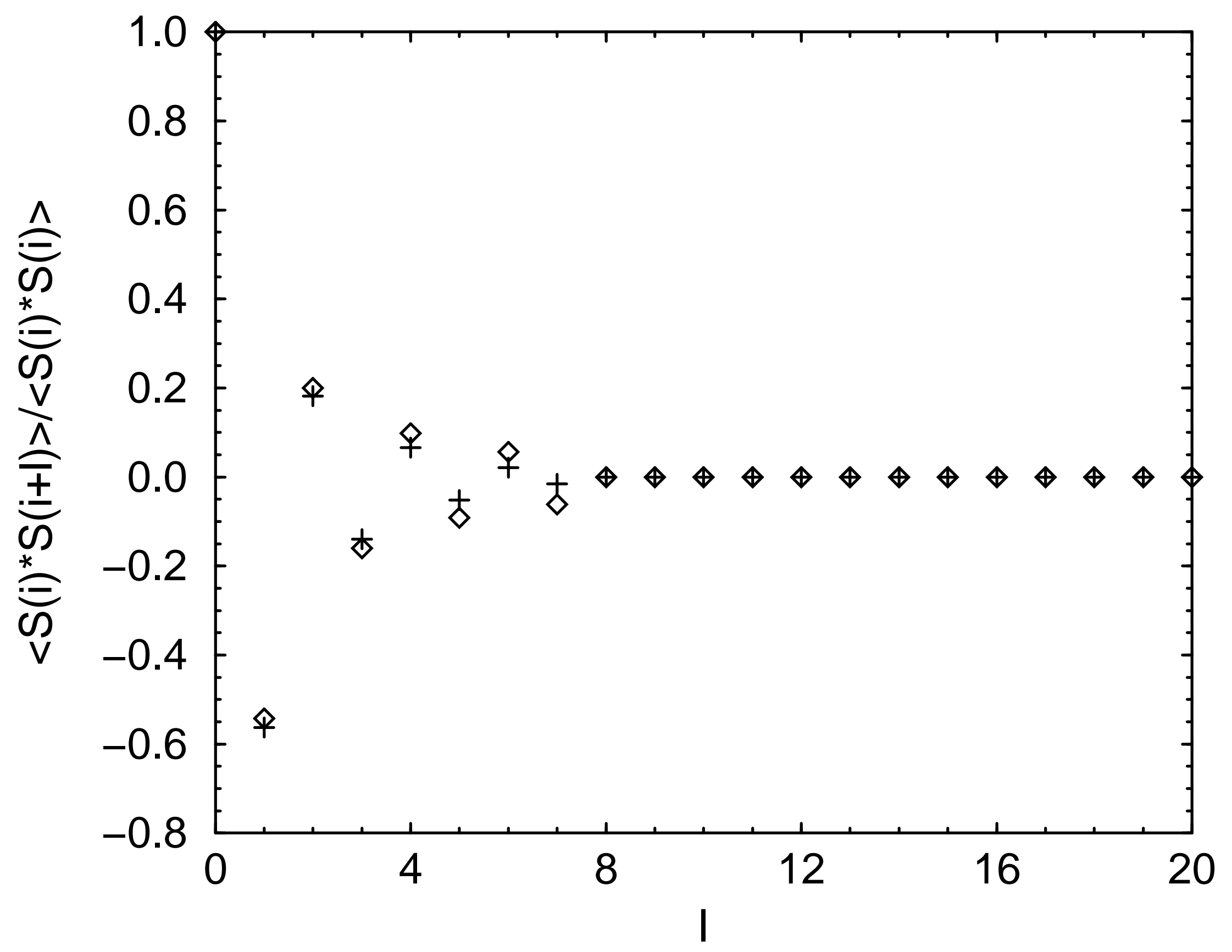




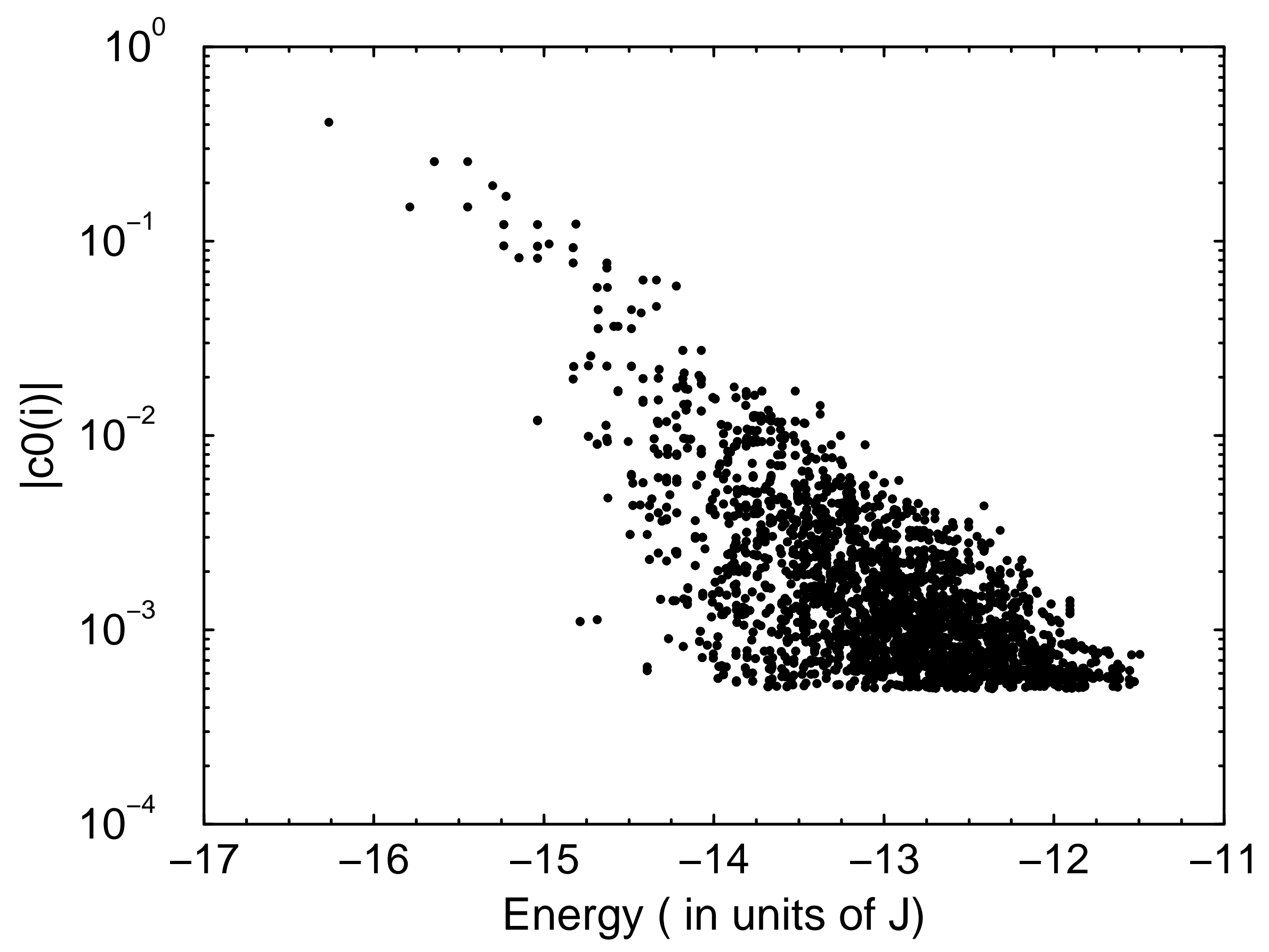

